\title{
Efeitos de um programa multiprofissional de tratamento da obesidade no ambiente aquático em adolescentes acompanhados ou não de suas mães
}

Effects of a multiprofessional obesity treatment program on the aquatic environment in adolescents with or without their mothers

Efectos de un programa multiprofesional de tratamiento de la obesidad en el medio acuático en adolescentes con o sin sus madres

Recebido: 12/01/2021 | Revisado: 13/01/2021 | Aceito: 13/01/2021 | Publicado: 14/01/2021

\author{
Mario Moreira Castilho \\ ORCID: https://orcid.org/0000-0002-4855-8236 \\ Universidade Estadual de Maringá, Brasil \\ E-mail: mmcastilho_1905@hotmail.com \\ Greice Westphal \\ ORCID: https://orcid.org/0000-0001-9107-0108 \\ Universidade Estadual de Maringá, Brasil \\ E-mail: greicewes@gmail.com \\ Igor Alisson Spagnol Pereira \\ ORCID: https://orcid.org/0000-0001-7340-3909 \\ Universidade Estadual de Maringá, Brasil \\ E-mail: igorspagnol2@hotmail.com \\ Fernando Malentaqui Martins \\ ORCID: https://orcid.org/0000-0003-1623-2183 \\ Universidade Estadual de Maringá, Brasil \\ E-mail: nandoesporte1@gmail.com \\ Karen Barros Meireles \\ ORCID: https://orcid.org/0000-0002-1757-7354 \\ Universidade Estadual de Maringá, Brasil \\ E-mail: kmeireles1@gmail.com \\ Nelson Nardo Junior \\ ORCID: https://orcid.org/0000-0002-6862-7868 \\ Universidade Estadual de Maringá, Brasil \\ E-mail: nnjunior@uem.br
}

\begin{abstract}
Resumo
Introdução: $\mathrm{O}$ aumento da obesidade tem sido registrado em todas as faixas etárias. Está pandemia, considerada a doença do século, atinge diretamente a saúde pública em todos os continentes e representa um desafio complexo da sociedade moderna. Objetivo: Comparar a perda de peso em adolescentes submetidos a um programa multiprofissional de tratamento da obesidade (PMTO) com e sem o acompanhamento de suas mães. Método: Foram selecionados 11 adolescentes, com idade entre 13 a 17 anos, de ambos os sexos, com sobrepeso ou obesidade. Todos os participantes assinaram o Termo de Consentimento Livre e Esclarecido (TCLE), antes de iniciar a sua participação. Todos indicaram ter disponibilidade e interesse para participar das intervenções com atividades no ambiente aquático, três vezes por semana, seguido de uma hora de orientações teóricas com profissionais das áreas de Educação Física, Nutrição e Psicologia. Foram realizadas avaliações antropométricas, seguindo procedimentos padronizados, as variáveis foram coletadas no pré-teste e pós-teste, após 16 semanas de um PMTO. Resultados: Os adolescentes apresentaram melhoras tanto no primeiro semestre quanto no segundo semestre, quando os mesmos foram acompanhados de suas mães. No entanto, no primeiro semestre foram observadas melhoras significativas $(\mathrm{p}<0,05) \mathrm{em}$ um maior número de variáveis, tais como a $\mathrm{CC}(\mathrm{p}=0,0389)$, gordura absoluta $(\mathrm{p}=0,0005)$ e percentual gordura $(\mathrm{p}=0,0009)$. No segundo semestre apresentaram melhoras significativas $(\mathrm{p}<0,05)$, no percentual de gordura e na FC de repouso. Conclusão: A participação no PMTO no ambiente aquático promoveu melhorias na aptidão física dos adolescentes, tanto quando eles foram acompanhados das mães, como na ausência das mesmas.
\end{abstract}

Palavras chave: Obesidade; Adolescentes; Mães; Ambiente Aquático.

\section{Abstract}

Introduction: The increase in obesity has been registered in all age groups. This pandemic, considered the disease of the century, directly affects public health on all continents and represents a complex challenge for modern society. Objective: To compare weight loss in adolescents undergoing a multiprofessional obesity treatment program (PMTO) 
with and without the monitoring of their mothers. Method: Eleven adolescents, aged between 13 and 17 years, of both sexes, were overweight or obese. All participants signed the Free and Informed Consent Form (ICF), before beginning their participation. All indicated having availability and interest to participate in interventions with activities in the aquatic environment, three times a week, followed by an hour of theoretical guidance with professionals in the areas of Physical Education, Nutrition and Psychology. Anthropometric assessments were carried out, following standardized procedures, the variables were collected in the pre-test and post-test, after 16 weeks of a PMTO. Results: The adolescents showed improvements both in the first semester and in the second semester, when they were accompanied by their mothers. However, in the first semester, significant improvements $(\mathrm{p}<0.05)$ were observed in a greater number of variables, such as WC $(p=0.0389)$, absolute fat $(p=0.0005)$ and fat percentage $(p=0.0009)$. In the second semester they showed significant improvements $(\mathrm{p}<0.05)$, in the percentage of fat and in resting HR. Conclusion: Participation in PMTO in the aquatic environment promoted improvements in the physical fitness of adolescents, both when they were accompanied by their mothers, and in their absence.

Keywords: Obesity; Adolescents; Mothers; Aquatic Environment.

\section{Resumen}

Introducción: El aumento de la obesidad se ha registrado en todos los grupos de edad. Esta pandemia, considerada la enfermedad del siglo, afecta directamente a la salud pública en todos los continentes y representa un desafío complejo para la sociedad moderna. Objetivo: Comparar la pérdida de peso en adolescentes sometidos a un programa multiprofesional de tratamiento de la obesidad (PMTO) con y sin seguimiento de sus madres. Método: Once adolescentes de 13 a 17 años de ambos sexos presentaban sobrepeso u obesidad. Todos los participantes firmaron el Formulario de Consentimiento Libre e Informado (ICF), antes de iniciar su participación. Todos manifestaron tener disponibilidad e interés para participar en intervenciones con actividades en el medio acuático, tres veces por semana, seguido de una hora de orientación teórica con profesionales en las áreas de Educación Física, Nutrición y Psicología. Se realizaron evaluaciones antropométricas, siguiendo procedimientos estandarizados, las variables se recolectaron en el pre-test y post-test, luego de 16 semanas de un PMTO. Resultados: Los adolescentes mostraron mejoras tanto en el primer semestre como en el segundo semestre, cuando fueron acompañados de sus madres. Sin embargo, en el primer semestre se observaron mejoras significativas $(p<0.05)$ en un mayor número de variables, como CC $(p=0.0389)$, grasa absoluta $(p=0.0005)$ y porcentaje de grasa $(p=0,0009)$. En el segundo semestre mostraron mejoras significativas ( $\mathrm{p}<0.05$ ), en el porcentaje de grasa y en la FC en reposo. Conclusión: La participación en PMTO en el medio acuático promovió mejoras en la condición física de los adolescentes, tanto cuando estaban acompañados de sus madres, como en su ausência.

Palabras clave: Obesidad; Adolescentes; Madres; Ambiente acuático.

\section{Introdução}

O crescimento da obesidade tem sido observado de forma generalizada, o que a torna um grave problema de saúde pública. No Brasil, nos Estados Unidos e em diversos outros países (Ng et al., 2014)(Ng et al., 2014). Também entre crianças e adolescentes a prevalência de sobrepeso e obesidade tem aumentado substancialmente (Lobstein et al., 2015; Ogden et al., 2016).

Este quadro vem despertando a preocupação de autoridades da área da saúde, pois essa população tem perfil de risco aumentado para doenças crônico não transmissíveis (DCNT) como o diabetes mellitus tipo 2, hipertensão arterial, distúrbios cardiometabólicos, digestivos, articulares e respiratórios. Além do risco aumentado de morte prematura (Christinelli et al., 2021; Kumar \& Kelly, 2017; Lobstein et al., 2015).

A obesidade na infância e adolescência é fortemente influenciada pela família (Florido et al., 2019). Entre os fatores familiares que favorecem o aparecimento da obesidade, Gomes e Nascimento (2015) mencionam: ausência da figura paterna pode determinar a gênese e manutenção da obesidade infantil; baixo nível de atividade física dos pais e, consequente, dos filhos; ausência de apoio para mudança de hábitos; baixo consumo de frutas e hortaliças; sobrepeso e obesidade de pais e mães; permissividade alimentar; excesso de uso da televisão ou de jogos eletrônicos; estigma social e não aceitação social da obesidade; baixa autoestima; e desestruturação familiar (Gomes \& Nascimento, 2015).

$\mathrm{O}$ aumento do número de pessoas com excesso de peso está relacionado às mudanças que têm ocorrido no estilo de vida. Os dois fatores que mais contribuíram para esse aumento são a ingestão de alimentos ricos em carboidratos e a redução do nível de atividades físicas e brincadeiras, que têm sido substituídos pela televisão, vídeo games e pelo computador (Malta et 
al., 2020). A diminuição ou mesmo a falta de exercícios físicos reduzem o gasto calórico entre crianças e adolescentes. Esse excesso de calorias passa a ser armazenado na forma de gordura, o que, a longo prazo, levará à obesidade. Pode-se ainda dizer que a obesidade está relacionada à falta de informações dos pais sobre prática de exercícios físicos regulares e hábitos saudáveis (Laux, 2015).

No estudo conduzido por Garasky et al. (2009) foram examinados os dados do Child Development Supplement of the Panel Study of Income Dynamics para crianças em duas faixas etárias: 5-11 e 12-17 anos de idade. Os resultados de um modelo de regressão com a categorização do peso infantil em peso saudável, excesso de peso e obesidade indicaram associações positivas entre uma série de estressores familiares como a falta de estimulação cognitiva e suporte emocional no domicílio entre crianças mais novas e problemas de saúde mental e física e tensão financeira na família entre crianças mais velhas e o sobrepeso e obesidade (Garasky et al., 2009).

Abrantes et al (2002) e Terres et al (2006) afirmam que, em crianças e adolescentes, as implicações da obesidade estão relacionadas ao aparecimento precoce de fatores de risco para doença arterial coronária, como diabetes mellitus tipo 2 , dislipidemia, hipertensão arterial sistêmica, bem como comprometimento postural, lesões musculoesqueléticas, problemas sociais e psicológicos (Abrantes et al., 2002; Terres et al., 2006).

Para cada $10 \%$ de aumento no peso corporal, há aumento na incidência de doenças coronarianas em aproximadamente 20\%, além da elevação no colesterol plasmático em torno de $12 \mathrm{mg} / \mathrm{dl}$. Isso está relacionado com a dislipidemia na obesidade, representada pela elevação do colesterol total, da lipoproteína de baixa densidade (LDL), dos triglicérides circulantes, e diminuição na lipoproteína de alta densidade (HDL). Esse risco pode se tornar mais acentuado quando o ganho de peso está acompanhado por redução na atividade física e alta ingestão de ácidos graxos saturados (Francischi et al., 2000).

Deste modo, o objetivo do estudo foi comparar a perda de peso de adolescentes entre 13 a 17 anos de ambos os sexos submetidos a um processo de intervenção com e sem o acompanhamento de suas mães.

\section{Metodologia}

Este estudo caracteriza-se como um ensaio clínico pragmático de intervenção (Patsopoulos, 2011). Os adolescentes selecionados, em sua maioria, pertenciam à rede pública de ensino e foram convidados a participar do estudo partir de divulgação na mídia local (TV, rádio, jornal) e meios eletrônicos (site, e-mail institucional, Facebook) o qual buscava adolescentes para a pesquisa, que atendessem aos seguintes critérios de inclusão: 1) ter idade entre 13 a 17 anos; 2) apresentar sobrepeso ou obesidade; 3) residir em Maringá, PR ou região metropolitana; 4) ter disponibilidade para participar integralmente das intervenções; 5) não estar realizando outro tratamento para obesidade (terapias, medicamentos, exercícios físicos regulares); 6) atestado médico (liberação médica); e 7) assinar o Termo de Consentimento Livre e Esclarecido (TCLE).

Este estudo foi realizado na Universidade Estadual de Maringá (UEM), Maringá, através do Núcleo de Estudos Multiprofissional da Obesidade (NEMO), que é um grupo de pesquisa vinculado ao Departamento de Educação Física (DEF) e ao Hospital Universitário Regional de Maringá (HUM), que realiza um programa denominado Programa Multiprofissional de Tratamento da Obesidade (PMTO), baseando em evidências sobre os riscos associados ao sedentarismo, sobrepeso e a obesidade, visando à melhora da qualidade de vida e a promoção da saúde dos participantes. Este PMTO teve duração de dois períodos distintos de 16 semanas. O programa contou com a participação de profissionais das áreas de Educação Física, Nutrição e Psicologia.

Foram incluídos no estudo adolescentes que apresentavam sobrepeso ou obesidade de grau I, II e III, de acordo com os pontos de corte de Cole e Lobstein (Cole \& Lobstein, 2012). Com o objetivo de comparar a perda de peso de adolescentes entre 13 a 17 anos de ambos os sexos submetidos a um PMTO com e sem o acompanhamento de suas mães em momentos 
distintos, participaram do estudo 11 adolescentes, no primeiro momento individual e no segundo momento, com a presença/participação de suas mães. A coleta de dados foi realizada em dois períodos distintos com pré e pós-teste. Entre os meses de março a junho foram coletados os dados apenas dos adolescentes, enquanto no período de agosto a dezembro de 2017, passou-se a ter a participação também das mães dos adolescentes.

Nesse estudo foi realizada a avaliação antropométrica (peso, altura, circunferências da cintura e IMC), a composição corporal (gordura absoluta, percentual de gordura e massa magra), foram mensurados também a pressão arterial e frequência cardíaca de repouso.

A massa corporal $(\mathrm{kg})$ foi mensurada por meio uma balança de tipo plataforma acoplada a um equipamento de bioimpedância elétrica de marca InBody Biospace, com resolução de 0,1 kg e capacidade máxima de $250 \mathrm{~kg}$. Os adolescentes foram orientados a comparecer no local da avaliação no período matutino, com roupas leves e retirar seus calçados e objetos que pudessem alterar o valor real da massa corporal.

A estatura (m) dos adolescentes foi medida por meio de um estadiômetro (Sanny) com precisão de $0,1 \mathrm{~cm}$ com campo de uso de $0,80 \mathrm{~cm}$ a 2,20m, seguindo os procedimentos recomendados por Lohman (Lohman et al., 1992). O IMC foi calculado através da divisão da massa corporal pela estatura ao quadrado e classificado de acordo com os valores propostos por Cole e Lobstein (Cole \& Lobstein, 2012). Foi mensurada a massa corporal (kg), estatura (m), índice de massa corporal - IMC $\left(\mathrm{kg} / \mathrm{m}^{2}\right)$.

A circunferência de cintura (CC) foi mensurada com uma trena antropométrica não extensiva de marca Sanny, com capacidade de medir até 2 metros e resolução de 0,1 cm (Lohman et al., 1992).

A avaliação da composição corporal foi realizada por meio do método da bioimpedância elétrica (BIA), por um equipamento de Marca Biospace-InBody Composition Analysers, (Coréia) modelo 520, em um ambiente controlado com temperatura entre 22 e $25{ }^{\circ} \mathrm{C}$. O avaliado posicionou-se fazendo contato direto dos pés com os eletrodos da plataforma (calcanhares e sola dos pés) e segurando firmemente com as mãos (eletrodos para contato com polegares e demais dedos), foram orientados a não se movimentar até o final da avaliação. Foi verificado para que as hastes (braços do equipamento) ficassem bem afastadas do corpo, de modo que os braços do avaliado não estivessem encostados no tronco, para garantir a diferenciação dos segmentos corporais durante a avaliação (Kyle et al., 2004).

As recomendações utilizadas para a realização da avaliação foram às propostas por Heyward (Heyward, 2001): urinar cerca de 30 min antes da avaliação; abster-se do consumo de álcool e bebidas cafeínas ao longo das últimas 48h; evitar esforços físicos vigorosos ao nas últimas $24 \mathrm{~h}$; não consumir diuréticos ao longo dos últimos sete dias; estar em jejum de alimentos sólidos e líquidos por mínimo de 2 horas; permanecer pelo menos 5 minutos em pé antes da avaliação. Para as adolescentes que se encontravam no período menstrual, foi redefinida a data de avaliação.

A aferição da frequência cardíaca e pressão arterial foram feitas utilizando o esfignomanômetro automático (Microlife, Argóvia, Suíça) e um manguito de capacidade apropriada para o tamanho do braço (AAP, 2004). O avaliado foi orientado para ficar sentado por 10 minutos (repouso), com as costas apoiada no encosto da cadeira, as pernas paralelas (semiextendidas) para facilitar uma melhor circulação. A pressão foi aferida no braço esquerdo, com a palma da mão voltada para cima, sobre uma mesa de superfície plana, o avaliador posicionou a braçadeira, realizou a marcação do instrumento na linha da artéria braquial e realizou três medidas com intervalo de três minutos entre elas, a primeira foi descartada e o valor final obtido foi o resultado da média das duas últimas (Bloch et al., 2016). O aparelho fornece os valores da Pressão Arterial Sistólica (PAS), Pressão Arterial Diastólica (PAD) e a Frequência Cardíaca (FC/Pulse).

No estudo foi aplicado um PMTO com duração de 16 semanas nos dois semestres entre as $18 \mathrm{~h} 30$ e $20 \mathrm{~h} 30$. As intervenções da área de Educação Física no ambiente aquático foram realizadas três vezes por semana (segundas, quartas e sextas-feiras) (Castilho et al., 2021). Além disso, uma aula teórica nas segundas feiras, executadas por educadores físicos, com 
a finalidade de orientar sobre a prática de atividade física e seus benefícios (com duração de uma hora) com objetivo de estimulá-los a praticar atividade física (Nardo Junior et al., 2018).

Foram realizadas reuniões mensais com os adolescentes e mães a fim de esclarecê-los acerca da importância da família no processo de mudança dos hábitos alimentares e de atividade física do adolescente. Além disso, no momento da reunião foram repassados individualmente os resultados parciais de cada participante no programa.

As intervenções práticas de exercícios no ambiente aquático ocorreram obedecendo uma série de orientações: no primeiro encontro todos os adolescentes e mães receberam informações quanto ao funcionamento das instalações físicas, dos procedimentos de segurança e sobre como fazer uso dos equipamentos de proteção individual como toucas, óculos e roupas de banho para a prática de hidroginástica e natação. As atividades aquáticas foram realizadas na piscina do Departamento de Educação Física da UEM, com dimensões de 25x12 metros, profundidade de 1,40 metros, separadas por 6 raias, a temperatura foi mantida entre $29^{\circ}$ e $31^{\circ}$ graus e o potencial hidrogeniônico permaneceu entre 7 a $8 \mathrm{pH}$. As intervenções foram ministradas por dois profissionais de Educação Física do grupo NEMO, que realizaram, predominantemente, atividades de hidroginástica, também se utilizando de exercícios de natação, com intensidade moderada, aumentada gradativamente ao longo do programa conforme padronização realizada dentro do próprio grupo em estudo prévio (Castilho et al., 2021; Lopera et al., 2016). Os exercícios começaram com alongamentos e as atividades de aquecimento como caminhadas e deslocamentos com alternância de frequência e intensidade moderada. Os equipamentos e materiais utilizados nas aulas foram: caixa de som, colchonetes, macarrão, halteres, bolas, pranchas e bastões.

A intervenção prática de natação foi dividida em quatro momentos com duração de 15 minutos cada um: exercícios de caminhada na água, exercícios com equipamentos para o domínio da natação, técnicas para o aprendizado da natação e atividades em pequenos e grandes grupos, com deslocamentos dentro da água e relaxamento final, conforme recomendações do ACSM (Castilho et al., 2021; Lopera et al., 2016).

Os procedimentos utilizados no desenvolvimento dessa pesquisa seguiram as regulamentações exigidas na Resolução $n^{\circ}$ 466/2012 do Conselho Nacional de Saúde sobre pesquisa envolvendo seres humanos. O protocolo de pesquisa foi previamente aprovado pelo Comitê de Ética em Pesquisa da Universidade Estadual de Maringá sob o parecer no 915.526/2014 e registrado na plataforma do Registro Brasileiro de Ensaios Clínicos (RBR-45ywtg).

Os dados obtidos foram digitados em planilhas do programa microsoft excel 2010 e as análises estatísticas foram processadas utilizando o pacote estatístico SPSS 22.0 (Andy Field, 2009). Foi realizado o teste t pareado para comparação de médias nos dois períodos avaliados, pré e pós teste, tanto no primeiro quanto no segundo momento. O nível de significância adotado nos testes foi de 5\%, ou seja, foram consideradas significativas as comparações cujo $p<0,05$.

\section{Resultados}

Foram avaliados 11 adolescentes com idade de 13 a 17 anos, de ambos os sexos, com média de peso de $91 \mathrm{~kg}$ no primeiro momento e $89,15 \mathrm{Kg}$ no segundo momento com avaliação do pré e pós-teste. Na tabela 1 estão apresentados os resultados das variáveis antropométricas, composição corporal e hemodinâmica. Houve diminuição significativa na CC $(\mathrm{p}=0,0389)$, Gord. Absoluta ( $\mathrm{p}=0,0005)$ e Percentual de Gordura $(\%)(\mathrm{p}=0,0009)$, embora tenha ocorrido a redução do peso, IMC, PAS, PAD e FC e o aumento da massa magra (MM), essas reduções não foram estatisticamente significativas. 
Tabela 1. Análise dos efeitos da Intervenção Multiprofissional nas variáveis antropométricas e composição corporal e Hemodinâmica no primeiro semestre (sem o acompanhamento das mães).

\begin{tabular}{|c|c|c|c|c|c|c|c|c|c|}
\hline \multirow{2}{*}{ Variáveis } & \multicolumn{4}{|c|}{$\begin{array}{c}\text { Pré-Intervenção } \\
(\mathrm{n}=11)\end{array}$} & \multicolumn{4}{|c|}{$\begin{array}{c}\text { Pós-Intervenção } \\
(\mathrm{n}=11)\end{array}$} & \multirow{2}{*}{$p$} \\
\hline & Média & Mínimo & Máximo & $\begin{array}{l}\text { Desvio } \\
\text { Padrão }\end{array}$ & Média & Mínimo & Máximo & $\begin{array}{l}\text { Desvio } \\
\text { Padrão }\end{array}$ & \\
\hline Peso $(\mathrm{Kg})$ & 91,0 & 69,2 & 129,7 & 18,4 & 88,3 & 65,0 & 123,9 & 17,5 & 0,0673 \\
\hline $\mathrm{IMC}\left(\mathrm{Kg} / \mathrm{m}^{2}\right)$ & 34,3 & 29,2 & 46,2 & 5,4 & 33,2 & 27,5 & 44,2 & 5,0 & 0,0650 \\
\hline $\mathrm{CC}(\mathrm{cm})$ & 97,8 & 89,3 & 112,0 & 8,6 & 94,5 & 81,0 & 107,5 & 7,5 & 0,0389* \\
\hline Gordura absoluta (Kg) & 42,6 & 27,2 & 68,2 & 12,2 & 38,5 & 21,7 & 63,1 & 11,9 & $0,0005 *$ \\
\hline Percentual de gordura $(\%)$ & 46,2 & 36,1 & 52,6 & 4,9 & 42,1 & 30,1 & 50,9 & 5,3 & $0,0009 *$ \\
\hline Massa magra (\%) & 45,6 & 33,7 & 58,3 & 7,2 & 46,8 & 33,3 & 57,4 & 7,0 & 0,0609 \\
\hline PAS (mmHg) & 114,5 & 95,0 & 135,0 & 10,8 & 113,4 & 90,0 & 135,0 & 11,7 & 0,8052 \\
\hline PAD (mmHg) & 69,4 & 56,0 & 78,0 & 7,7 & 67,4 & 57,0 & 76,0 & 5,7 & 0,4163 \\
\hline Frequência cardíaca (bpm) & 89,5 & 79,0 & 99,0 & 8,5 & 88,2 & 68,0 & 106,0 & 12,4 & 0,7642 \\
\hline
\end{tabular}

IMC: Índice de Massa Corporal. CC: Circunferência da cintura. *teste t pareado significativo considerando nível de significância de 5\%. Fonte: Autores.

Verificou-se que no segundo semestre conforme os dados estão apresentados na tabela 2, quando a intervenção foi realizada conjuntamente com filhos e mães que a perda de peso corporal foi inferior a perda ocorrida no primeiro semestre, quando apenas os filhos participaram da intervenção. O mesmo ocorrendo com a redução do IMC, circunferências, CC, CQ, RCQ, PAS, PAD e o aumento da massa magra (MM), sendo assim somente o percentual de gordura ( $\mathrm{p}=0,0341)$ teve diminuição significativa. Por outro lado, embora a FC mensurada no primeiro semestre não tenha apresentado alteração significativa, houve diminuição significativa na FC pré versus pós-teste dos adolescentes no segundo semestre.

Tabela 2. Análise dos efeitos da Intervenção Multiprofissional nas variáveis antropométricas e composição corporal e Hemodinâmica no segundo semestre (com o acompanhamento das mães).

\begin{tabular}{|c|c|c|c|c|c|c|c|c|c|}
\hline \multirow{2}{*}{ Variáveis } & \multicolumn{4}{|c|}{$\begin{array}{c}\text { Pré-Intervenção } \\
(n=11)(n=11)\end{array}$} & \multicolumn{4}{|c|}{$\begin{array}{c}\text { Pós-Intervenção } \\
(n=11)\end{array}$} & \multirow{2}{*}{$\mathbf{p}$} \\
\hline & Média & Mínimo & Máximo & $\begin{array}{l}\text { Desvio } \\
\text { Padrão }\end{array}$ & Média & Mínimo & Máximo & $\begin{array}{l}\text { Desvio } \\
\text { Padrão }\end{array}$ & \\
\hline Peso (Kg) & 89,15 & 64,70 & 129,20 & 18,70 & 88,35 & 65,00 & 122,40 & 17,05 & 0,3513 \\
\hline $\operatorname{IMC}\left(\mathrm{Kg} / \mathrm{m}^{2}\right)$ & 33,15 & 27,20 & 45,20 & 5,22 & 32,65 & 25,50 & 42,90 & 4,86 & 0,1554 \\
\hline $\mathrm{CC}(\mathrm{cm})$ & 94,36 & 82,00 & 108,00 & 9,21 & 91,19 & 78,70 & 105,50 & 7,79 & 0,0933 \\
\hline $\begin{array}{l}\text { Gordura absoluta } \\
(\mathrm{Kg})\end{array}$ & 38,85 & 21,50 & 66,60 & 12,73 & 37,05 & 16,70 & 58,90 & 12,15 & 0,0739 \\
\hline $\begin{array}{l}\text { Percentual de } \\
\text { gordura }(\%)\end{array}$ & 42,86 & 30,20 & 51,60 & 6,12 & 41,19 & 24,00 & 50,00 & 7,38 & $0,0341 *$ \\
\hline Massa magra $(\%)$ & 47,35 & 33,10 & 59,40 & 7,44 & 48,21 & 35,00 & 59,70 & 7,19 & 0,0524 \\
\hline PAS (mmHg) & 115,00 & 100,00 & 128,00 & 9,54 & 109,18 & 77,00 & 130,00 & 13,50 & 0,2648 \\
\hline PAD (mmHg) & 66,27 & 51,00 & 89,00 & 10,20 & 64,91 & 54,00 & 71,00 & 5,28 & 0,6842 \\
\hline $\begin{array}{l}\text { Frequência } \\
\text { cardíaca (bpm) }\end{array}$ & 88,64 & 61,00 & 103,00 & 11,72 & 67,82 & 51,00 & 105,00 & 15,86 & $0,0020 *$ \\
\hline
\end{tabular}

IMC: Índice de Massa Corporal. CC: Circunferência da cintura. *teste t pareado significativo considerando nível de significância de 5\%. Fonte: Autores.

No período de férias, entre os dois semestres, houve a interrupção das intervenções, isso pode ter contribuído para que os adolescentes voltassem a ganhar peso. O resultado do pré-teste do segundo semestre revela que o peso médio foi maior $(89,15 \mathrm{~kg})$, quando comparado com o pós-teste do primeiro semestre que foi de $(88,3 \mathrm{~kg})$. 


\section{Discussão}

A busca por uma melhor qualidade de vida, com a prática de atividades físicas, respeitando os limites individuais e com segurança, torna-se fundamental para o desenvolvimento de hábitos saudáveis e, é cada vez mais importante, não só para a saúde, mas, também para a aquisição de uma postura ativa e confiante frente a vida.

Neste estudo foram observadas reduções expressivas das medidas de circunferências da cintura, na gordura absoluta $(\%)$ e aumento de massa magra (\%) dos adolescentes incluídos no programa. Estes resultados foram verificados no primeiro semestre do programa, quando os adolescentes não estavam participando do PMTO juntamente com as suas mães.

O estudo também revelou que o programa continuou a contribuir para que os adolescentes, por meio da prática de exercícios físicos melhorassem, de forma estatisticamente significativa $(\mathrm{p}<0,05)$, o percentual de gordura $(\%)$ e a frequência cardíaca de repouso (bpm) no segundo semestre, quando os adolescentes realizaram as atividades em companhia com as suas mães.

De forma geral, os resultados obtidos em nosso estudo evidenciaram que a intervenção promoveu melhorias nos dois semestres, embora nem todas as variáveis investigadas tenham apresentado tal resultado. Destaca-se que, ao contrário do esperado, a companhia das mães não proporcionou a ampliação das respostas. Isso, no entanto, é compreensível, uma vez que os adolescentes já haviam realizado o programa durante um semestre e, portanto, alcançado mudanças significativas que não se perderam ao longo do segundo semestre do programa.

Em linha com o nosso estudo, o trabalho realizado por Yackobovitch-Gavan et al. (2018), que avaliaram os efeitos de intervenções familiares destinadas a pais e filhos na prevenção e tratamento da obesidade infantil, 247 crianças foram subdivididas em três grupos, sendo eles: Somente pais, pais e filhos e filhos sozinhos. Foi verificado que o grupo que reunia pais e filhos mostrou redução significativa do IMC. Com isso, concluíram que um programa de intervenção que enfoca tanto os pais quanto crianças trás efeitos positivos em curto e longo prazo para a redução do IMC (Yackobovitch-Gavan et al., 2018).

Em um estudo de acompanhamento, conduzido com 100 crianças, dos quatro aos sete anos de idade e respectivos pais, foi evidente que, quando o pai é fisicamente ativo, a propensão para a criança ser ativa é 3,4 vezes superior à de uma criança cujo pai é inativo. Por outro lado, quando a mãe é fisicamente ativa, a sua propensão é 2 vezes superior. Finalmente, quando ambos são fisicamente ativos, tal propensão é 7,2 vezes superior à de outras crianças cujos pais sejam fisicamente inativos (Moore et al., 1991).

As melhorias promovidas por programas multiprofissionais costumam atingir diversos parâmetros de avaliação. Assim, melhorias nos fatores de risco cardiovasculares geralmente estão relacionadas com o aumento da aptidão cardiorrespiratória (Castilho et al., 2020, 2021; Pereira et al., 2021; Westphal et al., 2020). Nesse sentido, Nassis, Psarra e Sidossis (2005) destacam que adolescentes que apresentam níveis de aptidão cardiorrespiratória mais elevados são os que possuem menor risco cardiovascular, independente do IMC (Nassis et al., 2005). Nesse sentido, a prática regular de exercícios proporcionada pelo PMTO e a orientação para que os participantes adotassem um estilo de vida ativo podem ter contribuído para a redução da gordura, aumento da massa magra e melhora na aptidão cardiorrespiratória.

Paes, Marins e Andreazzi (2015) afirmam que o exercício físico, independentemente do tipo, mostra-se capaz de promover adaptações positivas sobre a obesidade infantil, principalmente por atuar na restauração da homeostase celular e sistema cardiovascular, na melhoria da composição corporal e também aumento da taxa metabólica (Paes et al., 2015).

A participação das mães na prática dos exercícios no ambiente aquático juntamente com os filhos parece não ter exercido um efeito muito expressivo. No entanto, considera-se relevante a motivação que as mesmas produziram e o efeito dessa participação para ganhos nas próprias famílias em termos de promoção do controle de peso do adolescente, o que pode 
acarretar em benefícios de longo prazo, não só para os adolescentes, mas também para os seus familiares que, de alguma maneira, compartilharam consigo dessas experiências.

De acordo com Swift et al (2014) a participação em um programa de treinamento físico no tratamento da obesidade é importante para manter o controle da massa corporal em longo prazo (Swift et al., 2014). Benito et al (2015) afirmam que programas de treinamento físico são eficientes para melhorar as variáveis de composição corporal em relação à obesidade (Benito et al., 2015).

Programas multiprofissionais de tratamento da obesidade são, dessa forma, uma importante alternativa para o tratamento da obesidade e suas comorbidades (Christinelli et al., 2021; Pereira et al., 2021; Westphal et al., 2020). Em um estudo conduzido por Bianchini et al (2016), que avaliou o efeito de um PMTO em 97 adolescentes de ambos os sexos, com idades entre 10 e 18 anos, em 16 semanas de acompanhamento, sem a participação das mães, observaram reduções expressivas após a intervenção tanto em meninas quanto em meninos que apresentaram melhoras significativas no IMC, circunferência de cintura e quadril, massa gorda relativa e absoluta, massa magra, flexibilidade, força/resistência abdominal e aptidão cardiorrespiratória. Também foram identificadas melhoras nos parâmetros de aptidão física relacionadas à saúde, consumo máximo de oxigênio e resistência abdominal (Bianchini et al., 2016).

Em estudo realizado por Branco et al (2018), em adolescentes com sobrepeso ou obesidade, sem a presença das mães, comparou-se dois grupos de treinamento físico por um período de 12 semanas em um PMTO, o primeiro grupo trabalhou com exercícios envolvendo o próprio peso corporal (treinamento funcional) e o segundo grupo com a utilização de aparelhos de musculação. Foram observadas reduções do percentual de gordura corporal, massa gorda, circunferência da cintura e do quadril. Os autores concluíram que os dois métodos de treinamento físico foram significativos na melhora da composição corporal (Magnani Branco et al., 2018).

Lopera et al (2016) em estudo realizado com 67 crianças e adolescentes durante 16 semanas, divididos em três grupos de acordo com a idade $(\mathrm{G} 1=10-12 ; \mathrm{G} 2=13-15$ e G3 $=16-18$ anos), sem a presença dos pais, avaliaram as variáveis antropométricas, composição corporal e aptidão física. A intervenção de exercícios na água, esportes aquáticos e jogos realizados 3 vezes por semana com duração de 60 minutos a cada sessão promoveu alterações significativas nas variáveis: composição corporal e parâmetros antropométricos. Assim, concluíram que o PMTO de 16 semanas foi eficiente na melhoria da composição corporal de crianças e adolescentes (Lopera et al., 2016).

Já em estudo publicado em 2015, com o mesmo modelo de intervenção utilizado no presente trabalho, apenas com adolescentes, foram encontrados resultados satisfatórios quanto à melhoria da composição corporal e aptidão cardiorrespiratória (da Silva et al., 2015). Também foram reportadas melhorias em parâmetros hemodinâmicos, perfil lipídico e resistência à insulina (Bianchini et al., 2013), além de melhora na compulsão alimentar (Nardo Junior et al., 2016).

Um estudo longitudinal com uma amostra de 68 crianças e seus familiares, participantes do Programa "Fit Kids/Fit Families" realizado nos Estados Unidos, visando ajudar as crianças a manterem/diminuírem o IMC, aumentar a atividade física, diminuir atividades sedentárias, melhorar a autoestima e aumentar o conhecimento geral sobre comportamentos de vida saudáveis, verificou após 12 semanas de acompanhamento dos participantes do programa, que: os pais (96\%) e as crianças (81\%) demonstraram melhora em conhecimentos e atitudes em relação às mudanças de estilo de vida saudável, 59\% das crianças aumentaram a atividade física e $32 \%$ reduziram as atividades sedentárias, $81 \%$ apresentaram melhora enquanto $13 \%$ mantiveram seus índices de massa corporal e houve uma alta prevalência de melhora na autoestima (Joosse et al., 2008).

Com base nos efeitos positivos observados em estudos como o Fit Kids/Fit Families demonstrando a importância de programas voltados para o contexto familiar, bem como intervenções que utilizem o apoio dos pais na adoção de um estilo de vida saudável e ativo, sendo estes importantes preditores de comportamentos dos filhos (Seabra et al., 2008). Além disso, a Sociedade Brasileira de Pediatria reforça que ser fisicamente ativo todos os dias é importante para a promoção da saúde 
integral de crianças e adolescentes. É fundamental que as atividades sejam prazerosas e adequadas ao estado individual de crescimento e desenvolvimento da criança/adolescente (Sociedade Brasileira de Pediatria, 2017).

Evidências indicam que a atividade física durante a infância e a adolescência pode contribuir para o enfrentamento da obesidade ao menos por três caminhos: 1. a prática de atividade física na infância e adolescência auxilia no equilíbrio do balanço energético e, consequentemente, na prevenção e tratamento da obesidade e de doenças relacionadas à obesidade nesta fase da vida, 2. jovens ativos tendem a se tornar adultos ativos, aumentando o gasto energético durante todo o ciclo de vida e 3 . Jovens ativos possuem menor probabilidade de desenvolver obesidade e doenças relacionadas à obesidade na fase adulta (Sociedade Brasileira de Pediatria, 2017).

Diante de tais evidências, acredita-se que um PMTO, com a presença dos pais ou responsáveis ou somente com a participação dos adolescentes, pode ser uma ferramenta apropriada que contribui, de forma significativa, para melhorias na saúde e na qualidade de vida dos adolescentes.

\section{Conclusão}

O programa de intervenção com exercícios no ambiente aquático em dois momentos distintos, promoveu melhorias significativas em diversos parâmetros relacionados a saúde dos adolescentes, tais como na perda de peso e melhorias na composição corporal e parâmetros hemodinâmicos. Sugere-se, portanto, a realização de mais estudos com essa população como forma de ampliar a compreensão sobre os efeitos desse tipo de programa tanto nos adolescentes quanto em seus familiares, pois é possível que haja um efeito multiplicador que, se comprovado, potencializa o valor desse tipo de intervenção.

\section{Referências}

Abrantes, M. M., Lamounier, J. A., \& Colosimo, E. A. (2002). Prevalência de sobrepeso e obesidade em crianças e adolescentes das regiões Sudeste e Nordeste. Jornal de Pediatria, 78(4), 335-340. https://doi.org/10.1590/s0021-75572002000400014

Andy Field. (2009). Discovering Statistics using SPSS Statistics. SAGE Publications, 66, 822. http://www.amazon.com/Discovering-Statistics-using-IBMSPSS/dp/1446249182

Benito, P. J., Bermejo, L. M., Peinado, A. B., López-Plaza, B., Cupeiro, R., Szendrei, B., Calderón, F. J., Castro, E. A., \& Gómez-Candela, C. (2015). Change in weight and body composition in obese subjects following a hypocaloric diet plus different training programs or physical activity recommendations. Journal of Applied Physiology, 118(8), 1006-1013. https://doi.org/10.1152/japplphysiol.00928.2014

Bianchini, J. A. A., da Silva, D. F., Nardo, C. C. S., Carolino, I. D. R., Hernandes, F., \& Junior, N. N. (2013). Multidisciplinary therapy reduces risk factors for metabolic syndrome in obese adolescents. European Journal of Pediatrics, 172(2), 215-221. https://doi.org/10.1007/s00431-012-1865-7

Bianchini, J. A. A., Silva, D. F. DA, Lopera, C. A., Antonini, V. D. S., \& Nardo Junior, N. (2016). Intervenção multiprofissional melhora a aptidão física relacionada à saúde de adolescentes com maior efeito sobre as meninas em comparação aos meninos. Revista Brasileira de Educação Física e Esporte, 30(4), 1051-1059. https://doi.org/10.1590/1807-55092016000401051

Bloch, K. V., Klein, C. H., Szklo, M., Kuschnir, M. C. C., Abreu, G. de A., Barufaldi, L. A., Veiga, G. V. da, Schaan, B., Si lva, T. L. N. da, Moraes, A. J. P., Oliveira, A. M. A. de, Tavares, B. M., Magliano, E. da S., Oliveira, C. L. de, Cunha, C. de F., Giannini, D. T., Belfort, D. R., Santo, E. L., Leon, E. B. de, ... Goldberg, T. B. L. (2016). ERICA: prevalências de hipertensão arterial e obesidade em adolescentes brasileiros. Revista de Saúde Pública, 50(suppl 1), 1s-12s. http://www.scielo.br/scielo.php?script=sci_arttext\&pid=S0034-89102016000200306\&lng=en\&nrm=iso\&tlng=pt

Castilho, M. M., Westphal, G., Pereira, I. A. S., Martins, F. M., Bim, R. H., Thon, R. A., Moreira, V. F. R., Be vilaqua, C. A., Meireles, K. B., Okawa, R. T. P., \& Júnior, N. N. (2020). TESTE DE CAMINHADA DE 6 MINUTOS (TC6M) NA OBESIDADE SEVERA: CONSIDERAÇÕES. Revista Valore, 5(e-555).

Castilho, M. M., Westphal, G., Thon, R. A., Pereira, I. A. S., Martins, F. M., Amaral, M. F. do, Okawa, R. T. P., \& Nardo Junior, N. (2021). Efeitos de um programa multiprofissional de tratamento da obesidade no ambiente aquático em adultos com obesidade severa. Research, Society and Development, 10(1), e12910111636. https://doi.org/10.33448/rsd-v10i1.11636

Christinelli, H. C. B., Westphal, G., Nardo Junior, N., Borim, M. L. C., Costa, M. A. R., \& Fernandes, C. A. M. (2021). Nutritional status and body composition in individuals with overweight or obesity using usual and unusual indicators. Research, Society and Development, $10(1)$, e4910111339. https://doi.org/10.33448/rsd-v10i1.11339

Cole, T. J., \& Lobstein, T. (2012). Extended international (IOTF) body mass index cut-offs for thinness, overweight and obesity. Pediatric Obesity, 7(4), 284294. https://doi.org/10.1111/j.2047-6310.2012.00064.x

da Silva, D. F., Bianchini, J. A. A., Antonini, V. D. S., Barrero, C. A. L., Carolina, I. D. R., \& Junior, N. N. (2015). Effects of a multiprofessional program for 
treatment of obesity on feeding behavior and frequency in adolescents: differences between sexes. ConScientiae Saúde, 14(2), 246.

Florido, L. M. P., Mulaski, L. F. S., Hespanhol, M. B., Mendonça, R. C. S., Santos, T. C., Silva, W. A. S., \& Nunes, C. P. (2019). Combate À Obesidade: Estratégias Comportamentais E Alimentares. Revista Caderno de Medicina, 2(2), 80-89.

http://www.revista.unifeso.edu.br/index.php/cadernosdemedicinaunifeso/article/view/1367

Francischi, R. P. P. de, Pereira, L. O., Freitas, C. S., Klopfer, M., Santos, R. C., Vieira, P., \& Lancha júnior, A. H. (2000). Obesidade: atualização sobre sua etiologia, morbidade e tratamento. Revista de Nutrição, 13(1), 17-28. https://doi.org/10.1590/S1415-52732000000100003

Garasky, S., Stewart, S. D., Gundersen, C., Lohman, B. J., \& Eisenmann, J. C. (2009). Family stressors and child obesity. Social Science Research, 38(4), 755-766. https://doi.org/10.1016/j.ssresearch.2009.06.002

Gomes, M. J. M., \& Nascimento, E. G. C. do. (2015). AS MULTIFACETAS DO EXCESSO DE PESO NA CRIANÇA: UMA REVISÃO SISTEMÁTICA. Revista Brasileira de Ciências da Saúde - USCS, 13(45). https://doi.org/10.13037/ras.vol13n45.2667

Heyward, V. (2001). ASEP methods recommendation: Body composition assessment. Journal of Exercise Physiology Online, 4(4), 1-12.

Joosse, L., Stearns, M., Anderson, H., Hartlaub, P., \& Euclide, J. (2008). Fit kids/fit families: A report on a countywide effort to prom ote healthy behaviors Wisconsin Medical Journal, 107(5), 231-236.

Kumar, S., \& Kelly, A. S. (2017). Review of Childhood Obesity: From Epidemiology, Etiology, and Comorbidities to Clinical Assessment and Treatment. Mayo Clinic Proceedings, 92(2), 251-265. https://doi.org/10.1016/j.mayocp.2016.09.017

Kyle, U. G., Bosaeus, I., De Lorenzo, A. D., Deurenberg, P., Elia, M., Gomez, J. M., Heitmann, B. L., Kent-Smith, L., Melchior, J.-C., Pirlich, M., Scharfetter, H., Schols, A. M. W. J., \& Pichard, C. (2004). Bioelectrical impedance analysis--part I: review of principles and methods. Clinical Nutrition, 23(5), 12261243. http://www.sciencedirect.com/science/article/B6WCM-4D2FHYS-1/2/48048de1d2e629bf7411616e7702a083

Laux, R. C. (2015). 9 a 12 Anos De Idade De Uma Escola Pública Do Município De Chapecó, Sc. Portalperiodicos.Unoesc.Edu.Br, 209-214. https://portalperiodicos.unoesc.edu.br/acbs/article/view/6623

Lobstein, T., Jackson-Leach, R., Moodie, M. L., Hall, K. D., Gortmaker, S. L., Swinburn, B. A., James, W. P. T., Wang, Y., \& McPherson, K. (2015). Child and adolescent obesity: part of a bigger picture. The Lancet, 385(9986), 2510-2520. https://doi.org/10.1016/S0140-6736(14)61746-3

Lohman, T. J., Roache, A. F., \& Martorell, R. (1992). Anthropometric Standardization Reference Manual. Medicine \& Science in Sports \& Exercise, 24(8), 952. https://doi.org/10.1249/00005768-199208000-00020

Lopera, C. A., da Silva, D. F., Bianchini, J. A. A., Locateli, J. C., Moreira, A. C. T., Dada, R. P., Thivel, D., \& Nardo, N. (2016). Effect of water- versus landbased exercise training as a component of a multidisciplinary intervention program for overweight and obese adolescents. Physiology and Behavior, 165, 365373. https://doi.org/10.1016/j.physbeh.2016.08.019

Magnani Branco, B. H., Carvalho, I. Z., Garcia de Oliveira, H., Fanhani, A. P., Machado dos Santos, M. C., Pestillo de Oliveira, L., Macente Boni, S., \& Nardo, N. (2018). Effects of 2 Types of Resistance Training Models on Obese Adolescents' Body Composition, Cardiometabolic Risk, and Physical Fitness. Journal of Strength and Conditioning Research, 1. https://doi.org/10.1519/jsc.0000000000002877

Malta, D. C., Szwarcwald, C. L., Barros, M. B. de A., Gomes, C. S., Machado, Í. E., Souza Júnior, P. R. B. de, Romero, D. E., Lima, M. G., Damacena, G. N., Pina, M. de F., Freitas, M. I. de F., Werneck, A. O., Silva, D. R. P. da, Azevedo, L. O., \& Gracie, R. (2020). The COVID-19 Pandemic and changes in adult Brazilian lifestyles: a cross-sectional study, 2020. Epidemiologia e servicos de saude : revista do Sistema Unico de Saude do Brasil, $29(4)$, e2020407. https://doi.org/10.1590/S1679-49742020000400026

Moore, L. L., Lombardi, D. A., White, M. J., Campbell, J. L., Oliveria, S. A., \& Ellison, R. C. (1991). Influ ence of parents' physical activity levels on activity levels of young children. The Journal of Pediatrics, 118(2), 215-219. https://doi.org/10.1016/S0022-3476(05)80485-8

Nardo Junior, N., Bianchini, J. A. A., da Silva, D. F., Ferraro, Z. M., Lopera, C. A., \& Antonini, V. D. S. (2018). Building a response criterion for pediatric multidisciplinary obesity intervention success based on combined benefits. European Journal of Pediatrics, 177(6). https://doi.org/10.1007/s00431-018-3115-

Nardo Junior, N., Bolognese, M. A., Bianchini, J. A. A., Silva, D. F. da, Mendes, A. A., \& Dada, R. P. (2016). Efetividade de um programa multiprofissional de tratamento da obesidade em adolescentes: impacto sobre transtorno de compulsão alimentar periódica. Segurança Alimentar e Nutricional, 23(1), 807. https://doi.org/10.20396/san.v23i1.8635622

Nassis, G. P., Psarra, G., \& Sidossis, L. S. (2005). Central and total adiposity are lower in overweight and obese children with high cardiorespiratory fitness. European Journal of Clinical Nutrition, 59(1), 137-141. https://doi.org/10.1038/sj.ejcn.1602061

Ng, M., Fleming, T., Robinson, M., Thomson, B., Graetz, N., Margono, C., Mullany, E. C., Biryukov, S., Abbafati, C., Abera, S. F., Abraham, J. P., AbuRmeileh, N. M. E., Achoki, T., Albuhairan, F. S., Alemu, Z. A., Alfonso, R., Ali, M. K., Ali, R., Guzman, N. A., ... Gakidou, E. (2014). Global, regional, and national prevalence of overweight and obesity in children and adults during 1980-2013: A systematic analysis for the Global Burden of Disease Study 2013. The Lancet, 384(9945), 766-781. https://doi.org/10.1016/S0140-6736(14)60460-8

Ogden, C. L., Carroll, M. D., Lawman, H. G., Fryar, C. D., Kruszon-Moran, D., Kit, B. K., \& Flegal, K. M. (2016). Trends in Obesity Prevalence Among Children and Adolescents in the United States, 1988-1994 Through 2013-2014. JAMA, 315(21), 2292. https://doi.org/10.1001/jama.2016.6361

Paes, S. T., Marins, J. C. B., \& Andreazzi, A. E. (2015). Efeitos metabólicos do exercício físico na obesidade infantil: uma visão atual. Revista Paulista de Pediatria, 33(1), 122-129. https://doi.org/10.1016/j.rpped.2014.11.002

Patsopoulos, N. A. (2011). A pragmatic view on pragmatic trials. Dialogues in Clinical Neuroscience, 13(2), 217-224.

Pereira, I. A. S., Martins, F. M., Westphal, G., Castilho, M. M., \& Nardo Junior, N. (2021). Programas multiprofissionais de tratamento da obesidade com 
Research, Society and Development, v. 10, n. 1, e34010111929, 2021 (CC BY 4.0) | ISSN 2525-3409 | DOI: http://dx.doi.org/10.33448/rsd-v10i1.11929

metas de perda de peso definidas: uma revisão sistemática da literatura. Research, Society and Development, 10(1), e24710111779. https://doi.org/10.33448/rsd-v10i1.11779

Seabra, A. F., Mendonça, D. M., Thomis, M. A., Anjos, L. A., \& Maia, J. A. (2008). Determinantes biológicos e sócio-culturais associados à prática de atividade física de adolescentes. Cadernos de Saude Publica, 24(4), 721-736. https://doi.org/10.1590/S0102-311X2008000400002

Sociedade Brasileira de Pediatria. (2017). Promoção da Atividade Física na Infância e Adolescência. Promoção da Atividade Física na Infância e Adolescência: Manual de Orientação, 1, 1-14.

Swift, D. L., Johannsen, N. M., Lavie, C. J., Earnest, C. P., \& Church, T. S. (2014). The role of exercise and physical activity in weight loss and maintenance. Progress in Cardiovascular Diseases, 56(4), 441-447. https://doi.org/10.1016/j.pcad.2013.09.012

Terres, N. G., Pinheiro, R. T., Horta, B. L., Pinheiro, K. A. T., \& Horta, L. L. (2006). Prevalência e fatores associados ao sobrepeso e à obesidade em adolescentes. Revista de Saúde Pública, 40(4), 627-633. https://doi.org/10.1590/s0034-89102006000500011

Westphal, G., Baruki, S. B. S., Mori, T. A. de, Montebello, M. I. de L., \& Pazzianotto-Forti, E. M. (2020). Effects of Individualized Functional Training on the Physical Fitness of Women with Obesity. Lecturas: Educación Física y Deportes, 25(268), 61-75. https://doi.org/10.46642/efd.v25i268.2084

Yackobovitch-Gavan, M., Wolf Linhard, D., Nagelberg, N., Poraz, I., Shalitin, S., Phillip, M., \& Meyerovitch, J. (2018). Intervention for childhood obesity based on parents only or parents and child compared with follow-up alone. Pediatric Obesity, 13(11), 647-655. https://doi.org/10.1111/ijpo.12263 